[32] MagGi L. Ricerche sul controllo della freschezza e purezza del Latte. Thèse Lausanne, 1907. Institut d'Hygiène expérimentale et de parasitologie.

[33] Meter W., cité dans C. für Bakt. II. v. 51, p. 424. 1920.

[34] Meurer R. Z. für Fleish-und Milchhygiene, v. 35, p. 266. 1925.

[35] Ostertag R. Z. für Fleisch- und Milchhygiene, v. 20, p. 1. 1910.

[36] Prucha J., Harding, Weeter, Charmbers, eité dans le lait 1921, p. 123.

[37] Rodrigues A., cité dans Z. für Fleisch-und Milchhygiene, v. 36, p. 348. 1926.

[38] SchNeIder F., cité dans Le Lait 1923, p. 610.

[39] SKaR, O. Z. für Fleisch- und Milchhygiene, v. 23, p. 442. 1913.

[40] Skar O. Z. für Fleish-und Milchhygiene, v. 23, p. 301. 1913.

[41] Skar O. C. für Bakteriologie, II, v. 57, p. 326. 1922.

[42] Sмттн M.-D. The Jour. of Hygiene, v. 21, p. 139. 1922-1923.

[43] Supple G.-C. Whintng W.-A., Dawes C.-H., eité dans Le Lait, 1922, p. 477.

[44] Supple G.-C. et Floning G.-E., cité dans Le Lait,' 1925, p. 751.

[45] VroLl, Le Lait, 1921, p. 112.

[46] WeBster R.-O. Bulletin mensuel des renseignements agricoles et des maladies des plantes, 1920 , p. 450 .

[47] Mamelink S., eité dans Le Lait, 1921, p. 296.

[48] Neuk омм A., Schweizer Archiv für Tierheilkunde t. LXIX, fasc. 1. 1927

\title{
CONTRIBUTION A L'ÉTUDE DES LEUCOCYTES DU LAIT ET ESSAI DE LEUCOCYTO-DIAGNOSTIC DES DIFFÉRENTS ÉTATS PHYSIOLOGIQUES ET PATHOLOGIQUES DE CETTE SÉCRÉTION
}

\author{
par E. BOURGEOIS \\ Doeteur-Vétérinaire \\ Vétérinaire inspecteur sanitaire du Département de la Seine \\ (fin)
}

C. LAITs DE Mammites. - Au cours de l'étude des laits de rétention, nous avons été amené à parler des modifieations apportées par l'infection à la formule leucocytaire. Chaque fois, une élévation du pourcentage des polynucléaires neutrophiles fut constatée ; mais ces quelques observations nous ont paru insuffisantes pour l'établissement de conclusions indiscutables, aussi avons-nous demandé à l'étude des laits de mammite streptococcique les précisions indispensables nous permettant de caractériser l'infection dans la formule leucocytaire.

10 Mammite streptococcique contagieuse. - Nous avons eu la bonne fortune d'être appelé dans une étable de nourrisseur des environs de Paris pour procéder à l'éradication des animaux atteints de cette contagieuse affection qui, malgré les précautions hygiéniques et les soins médicaux éclairés d'un confrère distingué, faisait sans cesse de nouvelles victimes.

Pour ce faire, un échantillon de lait de chaque quartier a été prélevé vers la fin de la traite chez tous les animaux; pour chaque échantillon, la détermination du degré de catalase, la centrifugation, l'examen 
microscopique bactériologique et l'établissement de la formule leucocytaire ont été suecessivement effectués.

Cliniquement, l'affection ne faisait aucun doute : chez trois animaux le tarissement pathologique d'un ou de deux quartiers atrophiés fut constaté, et 9 eas de mammite répartis chez cinq animaux furent observés. Parfois, les quartiers atteints présentaient les classiques noyaux d'induration scléreuse au-dessus du trayon; d'autres fois, bien que le lait fut encore très contagifère, la mamelle était parfaitement normale à la palpation. Selon la variété du streptocoque, selon que l'infection était aigüe, subaigüe ou chronique, selon enfin que le prélèvement eût lieu au début, en pleine évolution ou à la fin de l'affection, les caractères physiques des échantillons et de leur culot de centrifugation furent très variables; le tableau VIII donne une idée de cette diversité. Tous les échantillons figurant à ce tableau furent trouvés anormaux après l'examen superficiel qui en fut fait ; tous accusèrent un degré de catalase catalase élevé ( 8 ccmes, 5 en moyenne); tous, à une exception près, furent trahis par leur goût salé ou par l'abondance et la viscosité de leur culot de centrifugation; mais 6 sur 9 , soit $66,66 \%$ environ, étaient d'apparence normale, ce qui montre une fois de plus la garantie illusoire qu'est en matière de lait l'aspect normal de ce produit.

Le earactère contagieux de l'affection, la palpation de certains quartiers malades, les caractères physiques du lait, les germes mis en évidence par le microscope, tout prouve qu'il s'agissait bien de la mammite contagieuse streptococcique de Nocard et Mollereav. L'examen microscopique a cependant permis d'observer deux variétés de streptocoques: les uns à chaînes relativement courtes et à gros grains, les autres à chaînes très longues et à grains fins justifiant l'expression de chevelu que beaucoup d'auteurs emploient pour décrire à la fois la gracilité et l'abondance des germes. Bien que nous n'ayons pas fait à cet égard d'étude bactériologique, nous inclinons à penser, après de nombreuses observations identiques, qu'il n'y a pas à proprement parler deux variétés de streptocoques déterminant la mammite contagieuse, mais que selon les conditions de culture dans la mamelle (degré d'acidité du milieu surtout), les germes apparaissent avec une morphologie différente. C'est là d'ailleurs une loi générale; dans les laboratoires, on observe journellement pour un même microbe, selon le milieu où il eultive, des formes très différentes. Parallèlement à nos examens leucocytaires et microbiens, nous regrettons de n'avoir pas eu la possibilité d'évaluer de façon très précise, par leur $\mathrm{pH}$ par exemple, l'acidité réelle des laits streptococciques étudiés. Peut-être eussions-nous pu établir une relation de cause à effet entre l'acidité et la variété microbienne observée. Puisse cette modeste suggestion ne pas passer inaperçue.

Quoi qu'il en soit, nous avons considéré les neuf échantillons de laits précités eomme provenant de quartiers atteints de mammite strepto- 
Tableau VIII

\begin{tabular}{|c|c|c|c|c|c|c|c|c|}
\hline \multirow{2}{*}{\multicolumn{2}{|c|}{$\begin{array}{c}\text { Dénomination } \\
\text { de } \\
\text { l'animal }\end{array}$}} & \multirow{3}{*}{$\begin{array}{l}\text { Quartier } \\
\text { atteint } \\
\text { A.D. }\end{array}$} & \multirow{2}{*}{$\begin{array}{l}\text { Aspect } \\
\quad \mathrm{du} \\
\text { liquide }\end{array}$} & \multirow{2}{*}{ Goût } & \multicolumn{2}{|c|}{$\begin{array}{l}\text { Culot de centrifugation pour } \\
20 \text { centimetres cubes }\end{array}$} & \multirow{2}{*}{$\begin{array}{l}\text { Degré de } \\
\text { catalase } \\
\text { exprimé } \\
\text { en c. cubes d'o }\end{array}$} & \multirow{2}{*}{$\begin{array}{c}\text { Variétés } \\
\text { de } \\
\text { Streptocoques }\end{array}$} \\
\hline & & & & & Quantité & caractères & & \\
\hline C & 1 & & jaunâtre grumeleux & salé & $1 \mathrm{cc} .1 / 4$ & $\begin{array}{l}\text { visqueux pu- } \\
\text { rulent }\end{array}$ & $9 \mathrm{cc}$. & $\begin{array}{c}\text { de } 10 \text { à } 20 \text { articles et } \\
\text { à gros grains }\end{array}$ \\
\hline C & 1 & P.G. & $\begin{array}{c}\text { jaunâtre sans } \\
\text { grumeaux }\end{array}$ & $d^{o}$ & 3 ce. $1 / 2$ & $d^{0}$ & 10 cc. 9 & $\begin{array}{c}\mathrm{d}^{\mathrm{o}} \\
\text { et à longues chaînes et } \\
\text { à grains fins }\end{array}$ \\
\hline C & 8 & A.D. & jaunâtre grumeleux & $\mathrm{d}^{\mathrm{o}}$ & $11 \mathrm{cc}$. & $\mathrm{d}^{0}$ & 9 cс. 8 & $\begin{array}{c}\text { à courtes chaînes et } \\
\text { gros grains }\end{array}$ \\
\hline C & 9 & P.G. & normal & $d^{o}$ & $1 / 4 \mathrm{ce}$ & visqueux & $9 \mathrm{cc}$. & $d^{o}$ \\
\hline C 1 & 12 & A. G. & $d^{0}$ & $d^{o}$ & $1 / 4 \mathrm{cc}$. & $d^{0}$ & $10 \mathrm{cc}$. & $\begin{array}{c}\text { à longues chaînes et à } \\
\text { grains fins }\end{array}$ \\
\hline C & 5 & A.G. & $d^{0}$ & $\begin{array}{l}\text { très peu } \\
\text { salé }\end{array}$ & peu abondant & peu visqueux & 6 cс. 5 & $d^{0}$ \\
\hline C & 5 & A.D. & $d^{o}$ & salé & $1 / 2 \mathrm{cc}$. & un peu visqueux & 10 cс. 9 & $\mathrm{~d}^{\mathrm{o}}$ \\
\hline C & 5 & P.G. & $d^{o}$ & normal & normal & visqueux & 4 cc. 9 & $d^{o}$ \\
\hline C & 5 & P.D. & $\mathrm{d}^{\mathrm{o}}$ & salé & do & peu visqueux & 6 cс. 8 & $\mathrm{~d}^{0}$ \\
\hline
\end{tabular}


coccique contagieuse. Quelles sont les caractéristiques leucocytaires de ces laits? La présence d'agents pathogènes dans les acini détermine un appel leucocytaire intense; aussi les leucocytes abondent-ils dans les frottis, et, de ce fait, la numération différentielle est plus rapidement effectuée ; elle est en outre beaucoup facilitée par la grande prédominance des polynucléaires, qui fait que l'apparition de leucocytes des autres catégories est, par champ, accidentelle. Un simple coup d'œil sur le tableau IX permet de se faire une idée très précise de la formule leucocytaire des laits de mammite streptococcique; ce qui frappe, c'est le pourcentage très élevé et à peu près identique des polynucléaires neutrophiles (80 à $87,8 \%$ ) et celui très faible des lymphocytes (1 à $7 \%$ ).

Une heureuse coïncidence a voulu que deux mois après avoir décelé une très légère rétention à l'examen du lait du quartier antérieur gauche de l'animal C. 5, nous constations sur ce même quartier une mammite streptococcique à son début; la comparaison des formules leucocytaires, avant et après l'infection, nous montrera le sens des modifications apportées à la formule leucocytaire par l'infection :

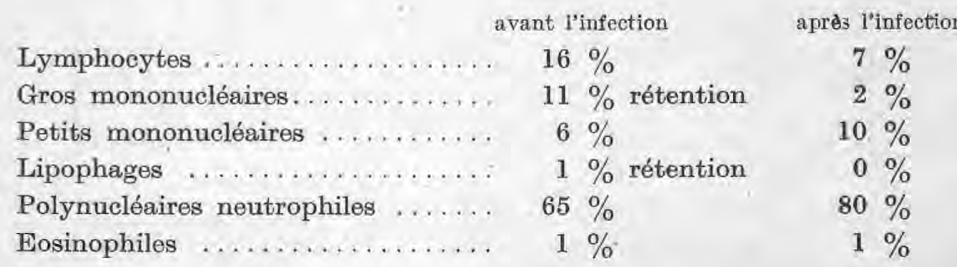

Bien que la formule leucocytaire avant l'infection fut celle d'un lait de légère rétention, non absolument normal par conséquent, et que le lait de ce quartier au deuxième prélèvement fut un de ceux pour lesquels l'infection streptococcique était la moins tapageuse (voir tableau VIII), nous voyons nettement que l'infection se traduit par une très sensible diminution des lymphocytes et une notable augmentation des polynucléaires neutrophiles, qui apparaissent dès lors comme les éléments microphages par excellence, défenseurs de l'organisme, rapidement à pied d'œuvre, grâce à leur mobilité amiboïde.

Pour la mammite streptococcique contagieuse, nous pouvons même préciser que le pourcentage des polynucléaires est de 85 en moyenne et celui des lymphocytes seulement de 3 . Le rapport $\frac{\text { mononucléaires }}{\text { polymucléaires }}$ varie dans cette affection de 0,13 à 0,25 , oscillant autour de 0,17 ; il est nettement différent de celui des laits normaux où il est en moyenne égal à 1 avec comme minimum 0,50 .

$2^{\circ}$ Mammites Banades. - Nous avons pu constater des modifications du même ordre que celles ci-dessus décrites, mais moins importantes, dans trois cas de légère infection par des cocci, qui n'ont pas été 
Tabléad IX

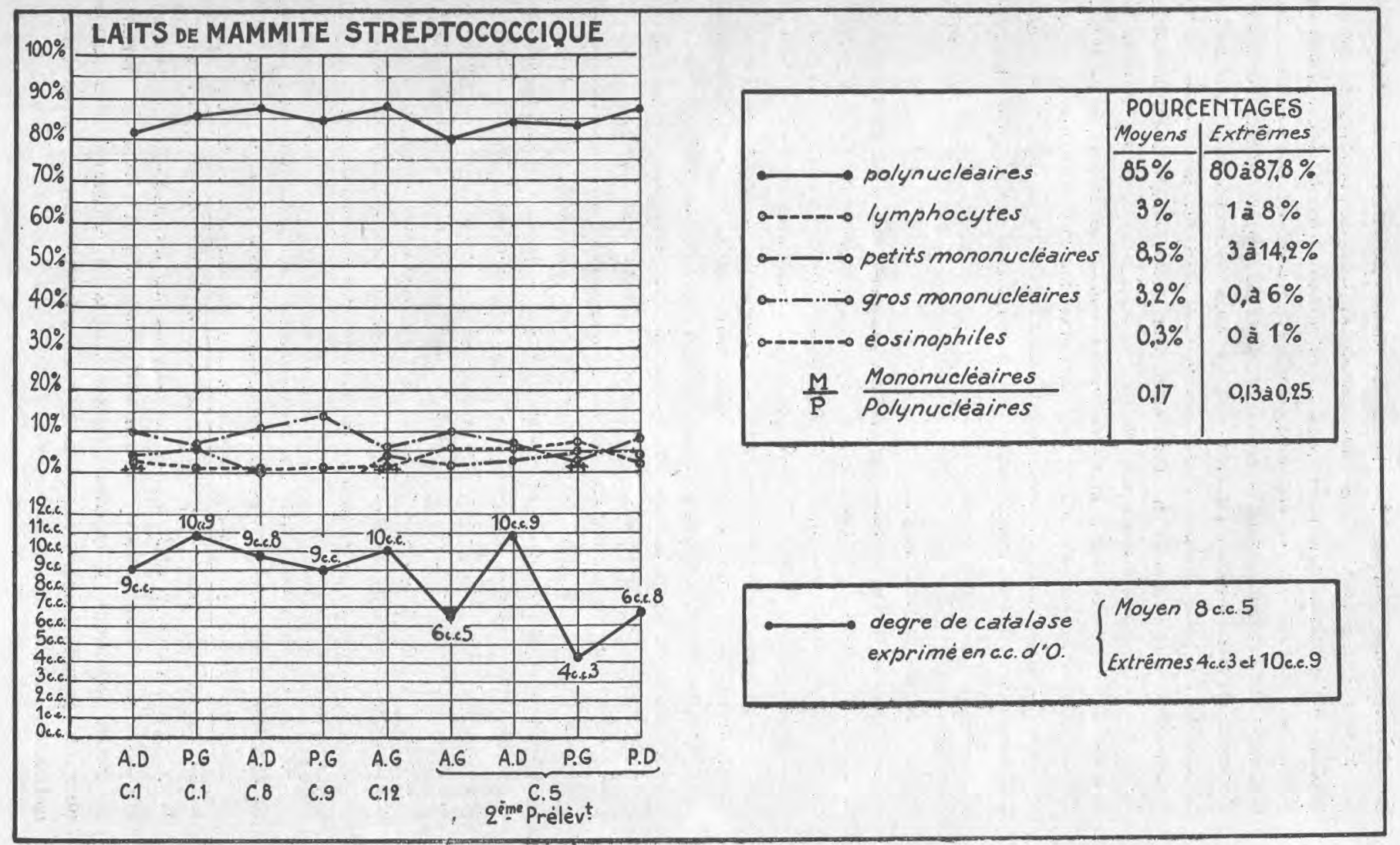


détermininés (voir tableau X). Dans ces trois eas (P.D. C 4; A.D. C 5 $\left(1^{\circ}\right.$ Prélevt) $^{t}$ et P.D. C.13), de tels germes étaient inclus dans le protoplasma de certains polynucléaires; l'infection, que ni les caractères physiques du lait, ni la centrifugation ne laissaient soupçonner, fut d'autre part confirmée par la détermination du degré catalasimétrique. Exprimé en centimètres cubes d'O dégagé, celui-ci fut en moyenne de 3,4, au lieu de 1,4 pour les laits normaux. Si l'on compare la formule leucocytaire de ces laits à celle des laits normaux, nous trouvons en moyenne $72 \%$ de polynucléaires au lieu de $48 \%$, et $2,5 \%$ de lymphocytes au lieu de $27 \%$; le sens des modifications est donc identique, mais d'une moins grande amplitude en ce qui concerne les polynucléaires, ce qui s'explique par ce fait qu'il s'agit ici d'une infection très légère non comparable à celle due au streptocoque de la mammite contagieuse. Le rapport $\frac{\mathrm{M}}{\mathrm{P}}$ est en moyenne de 0,36 au lieu de 1 , avec comme minimum 0,50 pour les laits normaux.

Il va sans dire que pour la grande majorité des cas étudiés, nous nous sommes trouvé el présence d'infections polymicrobiennes à germes indéterminés (streptocoques, staphylocoques, cocci, bâtonnets, bactéries, etc.). Selon le degré de ces infections, selon le moment de leur évolution, les caractères ordinaires des laits de mammite apparurent plus ou moins nettement. Parfois, il fut même difficile de déceler l'infection; mais chaque fois que la phagocytose de germes fut corstatée, la formule leucocytaire traduisit nettement l'infection par son pourcentage élevé de polynucléaires (70 à $98 \%$ ). C'est dire l'extrême sensibitité de l'examen leucocytaire différentiel comme moyen de diagnostic des laits anormaux.

Nous avions conçu le projet d'établir le diagnostic différentiel des infections streptococciques et bacillaires par l'examen comparatif des formules leucocytaires, complété au besoin par celui des figures nucléaires des neutrophiles. La chance ne nous a pas favorisé, les quelques cas de laits tuberculeux que nous pûmes nous procurer au marché de la Villette sur des laitières réformées par la boucherie étaient en outre souillés par d'autres germes et en particulier par des streptocoques; dans ces conditions, tout essai de diagnostic différentiel devenait impossible,

Malgré cette lacune, que les circonstances ne nous ont pas permis de combler, nous croyons avoir suffisamment montré que.l'examen différentiel des leucocytes constituait un moyen de diagnostic des laits anormaux, capable de rendre de précieux services dans les cas les plus douteux. Nous synthétiserons sous forme de conclusions les résultats acquis au cours de ce travail ; mais, auparavant, nous tenons, dans un dernier. et court chapitre, à attirer l'attention sur les rapports existant entre le pourcentage des polynucléaires neutrophiles et le degré de catalase. 
PLANCHE I
A. - LAIT NORMAL

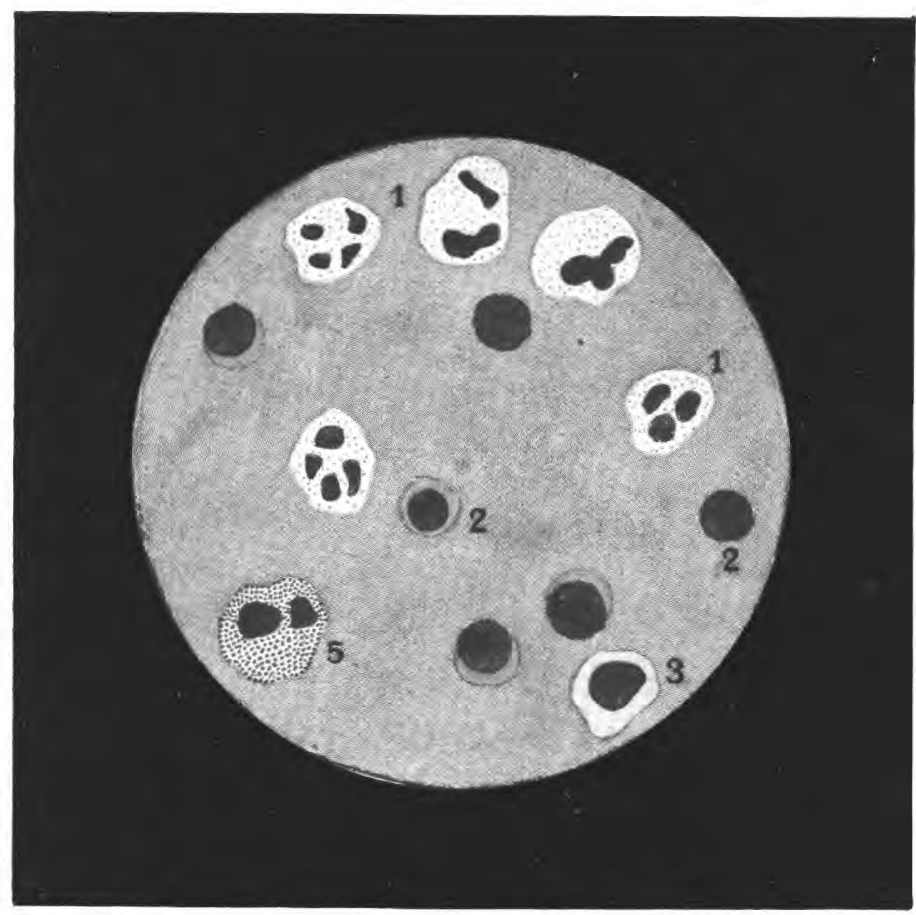

B. - LAIT DE MAMMITE STREPTOCOCCIQUE

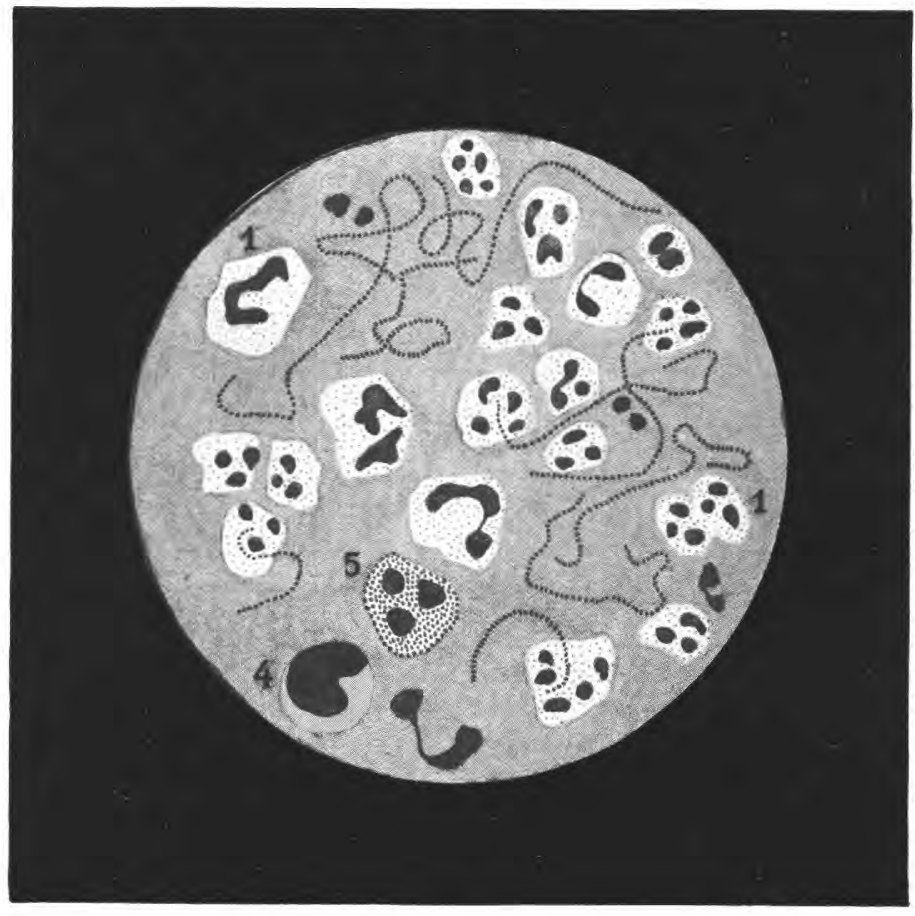

1. Polynucléaires neutrophiles

4. Gros mononucléaires 
C. - LAIT DE REtention

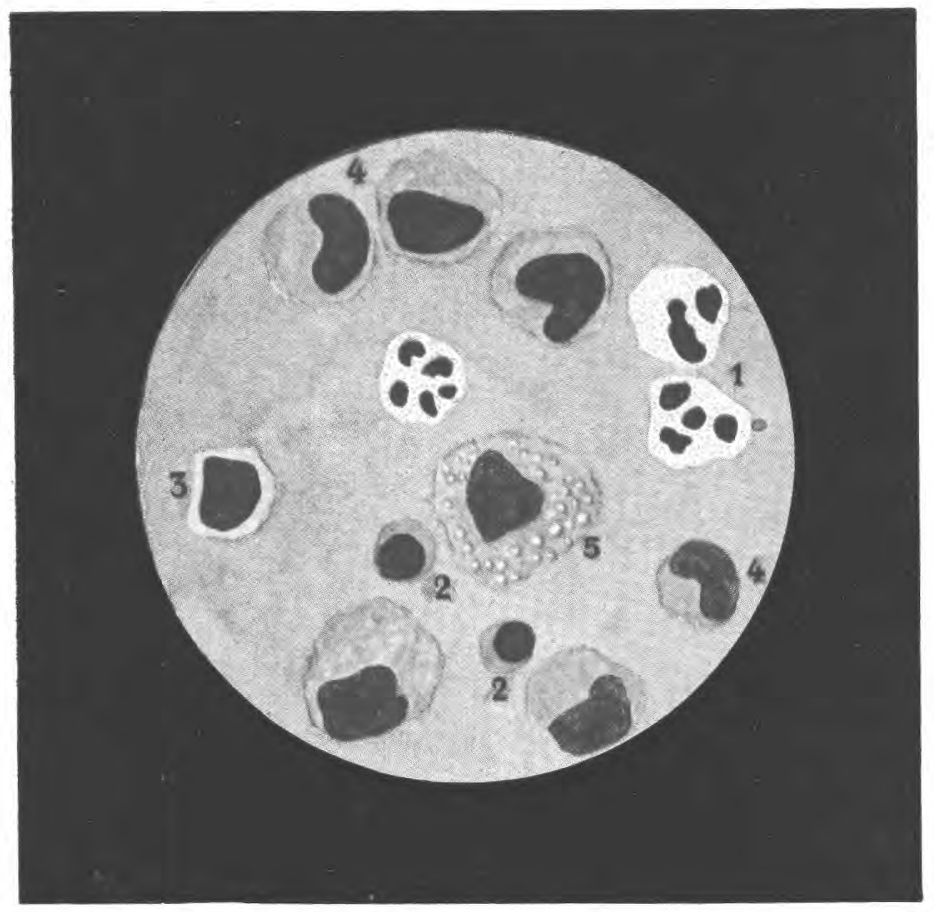

D. - colostrum

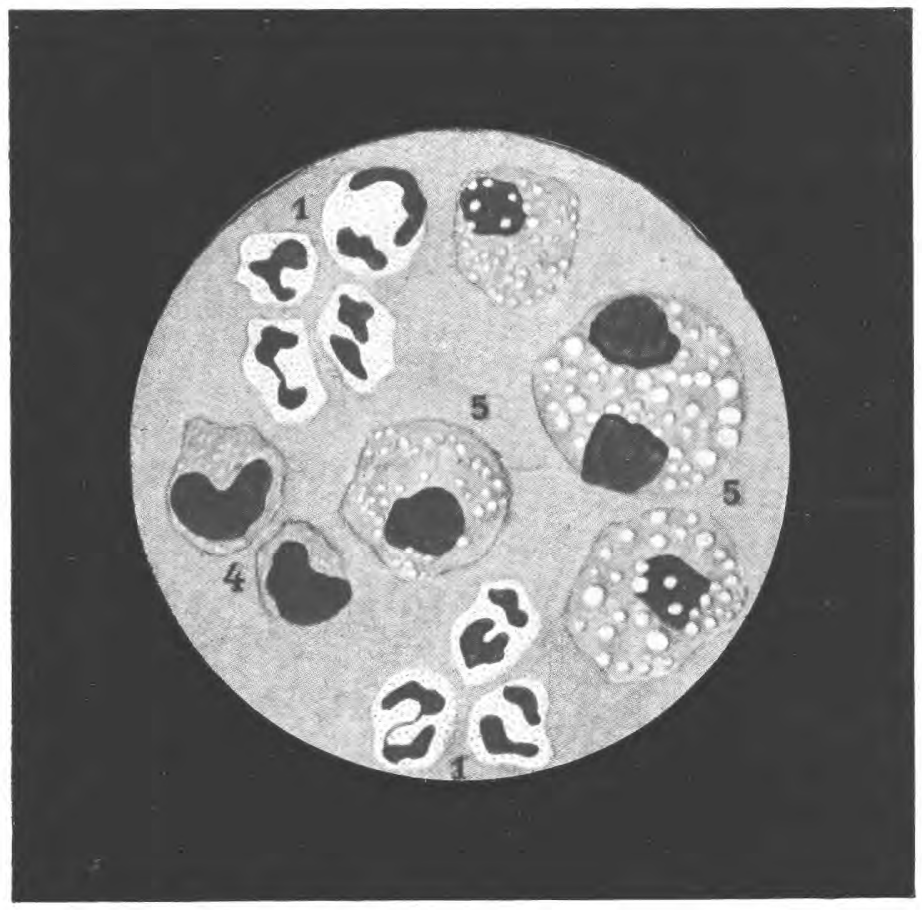

1. Polynucléaires neutrophiles

\section{Gros mononucléaires}





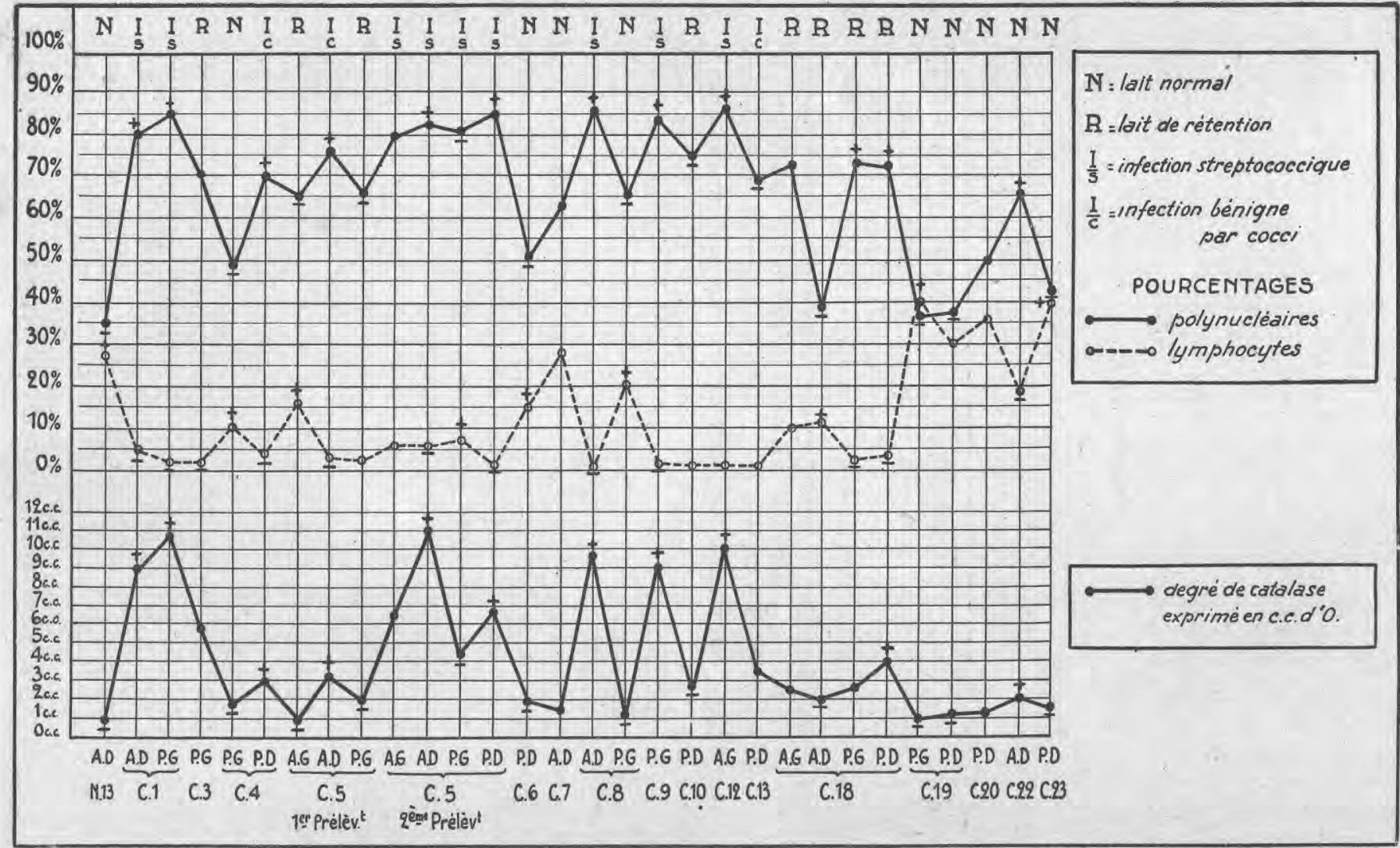

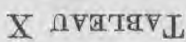




\section{$5^{\circ}$ Degré de catalase et pourcentage des polynucléaires.}

Nous avons été frappé du parallélisme, constaté dans 90 pour cent des cas, entre le pourcentage des polynucléaires et le degré de catalase. Si nous juxtaposons (ce que nous avons réalisé dans le tableau $\mathbf{X}$ ), les courbes représentatives de ces deux données, nous observons une analogie entre les deux tracés : aux points élevés de la courbe donnant les degrés de catalase correspondent les points élevés de celle donnant le pourcentage des polynucléaires, et inversement. Poussant plus loin la comparaison entre le degré de catalase et le pourcentage des autres catégories de leucocytes, nous avons fait, concernant les lymphocytes, des observations diamétralement opposées. Polynucléaires et lymphocytes s'opposent donc très nettement à tous points de vue; ceci confirme notre manière de voir: les lymphocytes sont de simples éléments de filtration, sans aucun rôle défensif, non producteurs de catalase, semblet-il, puisqu'à leurs pourcentages les plus élevés correspondent les degrés catalasimétriques les plus faibles. Au contraire, les polynucléaires, éléments microphages par excellence, seraient les principaux producteurs de cette diastase, puisqu'à un lait très riche en catalase, correspond toujours un pourcentage très élevé de ces granulocytes.

La mise en évidence d'une relation étroite entre le pourcentage des polynucléaires et le degré de catalase semble être à première vue un argument d'une certaine valeur pour les auteurs qui voient dans les leucocytes les grands producteurs de cette diastase; mais, en vérité, cette constatation n'infirme en rien les assertions de ceux qui considèrent la catalase surtout comme une diastase microbienne. En effet, lorsque le pourcentage des polynucléaires est anormal, les microbes, qui ont déterminé la venue en masse de ces leucocytes dans les acini, sont aussi très nombreux et à wn degré catalasimétrique élevé correspondent à la fois un pourcentage élevé de polynucléaires et un grand nombre de microbes.

Quoi qu'il en soit, ces quelques remarques méritaient d'être signalées ; si elles ne permettent pas encore une solution du problème de la catalase, elles contribueront peut-être, pour leur part, à jalonner la route qui y conduira.

\section{CONCLUSIONS}

La répression des fraudes en matière de lait repose sur un examen purement chimique; elle ne saurait en aucun cas concourir à l'application du décret du 25 mars 1924, concernant le commerce du lait et des produits de laiterie.

Un contrôle sanitaire du lait à la production est indispensable; en dehors de la vérification des conditions hygiéniques générales de logement et de nourriture, l'examen clinique des animaux et en particulier de leur mamelle s'impose ; de plus, l'examen du lait quartier par quartier, 
pour se rendre compte de son état à la sortie de la glande, apparaît aussi comme une nécessité.

Pour ce faire, et.lorsque les moyens ordinaires de diagnostic ne lui auront pas permis de se prononcer, le futur contrôleur sanitaire ne devra pas hésiter à recourir à des méthodes plus longues, peut-être plus délicates, mais aussi plus sûres. II devra bien se pénétrer de cette idée que «la mamelle vraiment saine est l'exception, alors qu'au contraire la mamelle infectée est presque la règle " (Ch. Porcher). Le plus souvent cette infection revêt une forme, insidieuse que ni l'examen clinique, ni l'examen superficiel du lait lui-même ne peuvent mettre en évidence. Dans la grande majorité des cas, le laboratoire seul permettra à l'hygiéniste, en présence d'une glande saine, de savoir si le lait qu'elle sécrète est réellement normal.

Parmi les moyens efficaces qu'offre le laboratoire, le leucocytodiagnostic est particulièrement à reeommander. Venu du sang et peut-être aussi directement de la lymphe, les leucocytes du lait sont, selon les cas, de simples éléments de filtration ou au contraire de vigilants défenseurs de la glande. Il est donc très rationnel que l'on ait considéré comme un moyen de contrôle.sanitaire du lait, d'une part, l'évaluation de la quantité de ses leucocytes, d'autre part, l'établissement, de sa formule leucocytaire, pour savoir s'il y a prédominance des éléments de pure filtration (laits normaux) ou des éléments plus spécialement accourus pour la défense de l'organisme (rétention et infection).

Nous avons montré au cours de ce travail que l'appréciation quantitative du contenu cellulaire n'était pas chose facile et que même le serait-elle, elle ne pouvait pas le plus souvent entraîner à elle seule la conviction qu'un lait était bon ou mauvais; des laits à faible contenu cellulaire ont été reconnus infectés et au contraire des laits à contenu cellulaire abondant ont été jugés normaux. Il est donc plus important de savoir QUELLES cellules se trouvent dans le lait suspect que de savoir COMBIEN il en contient.

Nous avons pensé que pour juger de la qualité sanitaire d'un lait, seuls les leucocytes étaient à considérer, et la formule leucocytaire nous est apparue COMME LA PHYSIONOMTE DE CE LAIT. Grâce à elle, on verra immédiatement si les éléments de pure filtration (lymphocytes), en proportion respectable dans les laits normaux, n'ont pas à peu près disparu dans la foule débordante et très dense des éléments de défense ; s'il en est ainsi, l'examen de ces éléments les plus abondants permettra de savoir le but de cette défense. Si c'est contre la rétention, les macrophages seront en grand nombre, déjà plus ou moins gorgés de globules gras ; si c'est contre une invasion microbienne, les polynucléaires, microphages par excellence, auront envahi en rangs serrés les acini.

Pour que cette méthode ait toute sa valeur, il est indispensable que son usage soit limité à l'examen des laits de chaque quartier prélevés 
séparément. Chaque quartier de mamelle produit un lait quantitativement et qualitativement sous la dépendance, au point de vue leucocytaire, de son état physiologique ou pathologique; aussi ne faut-il pas demander à la formule leucocytaire d'un lait de mélange des indications précises. Cette inaptitude de la méthode préconisée comme moyen de diagnostic d'une fourniture de lait est certaine; mais tel n'est pas le but de ce travail; nous avons seulement voulu donner au futur contrôleur A LA PRODUCTION la possibilité de vaincre son hésitation lorsque les autres moyens de diagnostic ne lui auront pas permis de se prononcer en toute certitude sur la qualité sanitaire du lait d'un quartier. Economiquement et sanitairement, il est d'ailleurs du plus grand intérêt de savoir sur quel quartier de la mamelle portent la rétention ou l'infection; nous pensons que la délicate sensibilité de notre méthode d'examen permettra le plus souvent de satisfaire cette curiosité.

Nos recherches personnelles nous ont amené aux conclusions suivantes :

$1^{0} \mathrm{La}$ formule leucoeytaire d'un lait normal est très voisine de celle du sang; la proportion des lymphocytes, éléments de filtration, venus directement de la lymphe et du sang, y est toujours plus élevée. Ce sont les laits normaux qui ont la plus grande proportion de lymphocytes et dans lesquels on observe le moins de polynucléaires. Le rapport M. (mononucléaires)

$\frac{\mathrm{M} \text { (mononucléaires) }}{\mathrm{P} \text { (polynucléaires) }}$ est en moyenne sensiblement égal à̀ l'unité, en tout cas jamais inférieur à 0,50 .

Les leucocytes y sont rares et le degré de catalase est toujours faible. Les laits qui présentent ces caractères sont l'exception. Cette constatation, qui confirme les résultats établis par de nombreux examens bactériologiques des laits, n'est malheureusement pas faite pour encourager les partisans de la consommation du lait cru, propre et sain, puisqu'elle tend à démontrer que quatre-vingt-dix fois sur cent les laits sont déjà infectés dans la mamelle. Il n'en reste pas moins du plus grand intérêt de pouvoir différencier à leur sortie de la mamelle les laits sains, indispensables à l'alimentation d'une certaine catégorie de consommateurs.

$2^{\circ}$ La rétention se traduit dans le contenu leucocytaire par l'apparition d'une proportion importante de gros mononucléaires lipophages. Si elle est légère, les gros mononucléaires chargés de graisse sont rares ; si, au contraire, elle est prononcée, les éléments gras sont en plus grand nombre que ceux dépourvus de graisse. Notons en passant que la rétention, aussitôt qu'elle devient importante, s'accompagne neuf fois sur dix d'infection; ces deux phénomènes, rétention et infection, s'appellent réciproquement. Dans ce cas, aux éléments caractéristiques de la rétention (gros macrophages), s'ajoutent jusqu'à prédominance très marquée, les polynucléaires mierophages. 
$3^{\circ}$ Le colostrum a une formule leucocytaire de lait de rétention avec cette particularité toutefois que les éléments de rétention sont à un suprême degré de différenciation; les lipophages sont littéralement bourrés de globules gras, et plus ou moins en voie de dégénérescence; le noyau disparaît alors partiellement et quelquefois même totalement ; le corpuseule typique de DonNé est constitué.

$4^{0}$ Dès que l'infection survient, le pourcentage des lymphoeytes tombe à 3 en moyenne, et la presque totalité des leucocytes (très nombreux dans de telles conditions) est constituée par les polynucléaires neutrophiles dont la proportion atteint 70 à $75 \%$ dans les infections légères par cocci, 80 à $85 \%$ dans la mammite streptococcique contagieuse et jusqu'à $98 \%$ dans certaines infections polymicrobiennes même insidieuses. Le tableau XI résume les résultats acquis.

Nous avons en outre essayé d'établir la formule nucléaire de quelques laits sains et infectés ; nos observations ne sont pas assez nombreuses ni assez nettes, pour nous permettre des conclusions fermes à cet égard. Disons seulement qu'il semble que les polynucléaires à 3 noyaux sont les plus nombreux dans les deux cas; mais alors que ceux à 4 et 5 noyaux sont relativement abondants dans les laits normaux, ce sont ceux à 2 noyaux qui oceupent la deuxième place dans les laits infectés. Peut-être y a-t-il dans l'utilisation de ces précisions un moyen de diagnostic différentiel de certaines affections mammaires.

50 La comparaison au cours de nos recherches du degré de catalase des laits avec le pourcentage des différents leucocytes, nous a permis de constater une relation très nette entre le degré de catalase et le pourcentage des polynucléaires, ce qui nous autorise à penser que de tous les leucocytes, les polynucléaires sont surtout producteurs de cette diastase.

Telles sont rapidement résumées les conclusions auxquelles nous avons abouti.

La technique que nous avons adoptée pour nos recherches est délicate, compliquée même, mais il était indispensable qu'elle donnât toute garantie. Dans la pratique, on pourra sans inconvénient simplifier la méthode de coloration; point n'est besoin de reeourir en effet à une coloration différentielle des éosinophiles, puisque ces leucocytes spéciaux, uniquement en rapport avec l'état parasitaire de l'organisme, ne semblent pas avoir dans le lait de signification sanitaire.

Nous nous rendons parfaitement compte de ce que ce travail a d'incomplet; on pourra regretter que nous n'ayons pas fixé les différentes formules leucocytaires correspondant aux principales infections de la mamelle, comme nous l'avons fait pour la mammite streptococcique contagieuse par exenuple. Il faut bien dire d'abord que l'expérimentation, base de telles recherches, nous fût matériellement impossible étant donné le prix actuel des animaux d'expérience, et que, d'autre part, il est peu 
Tableau XI

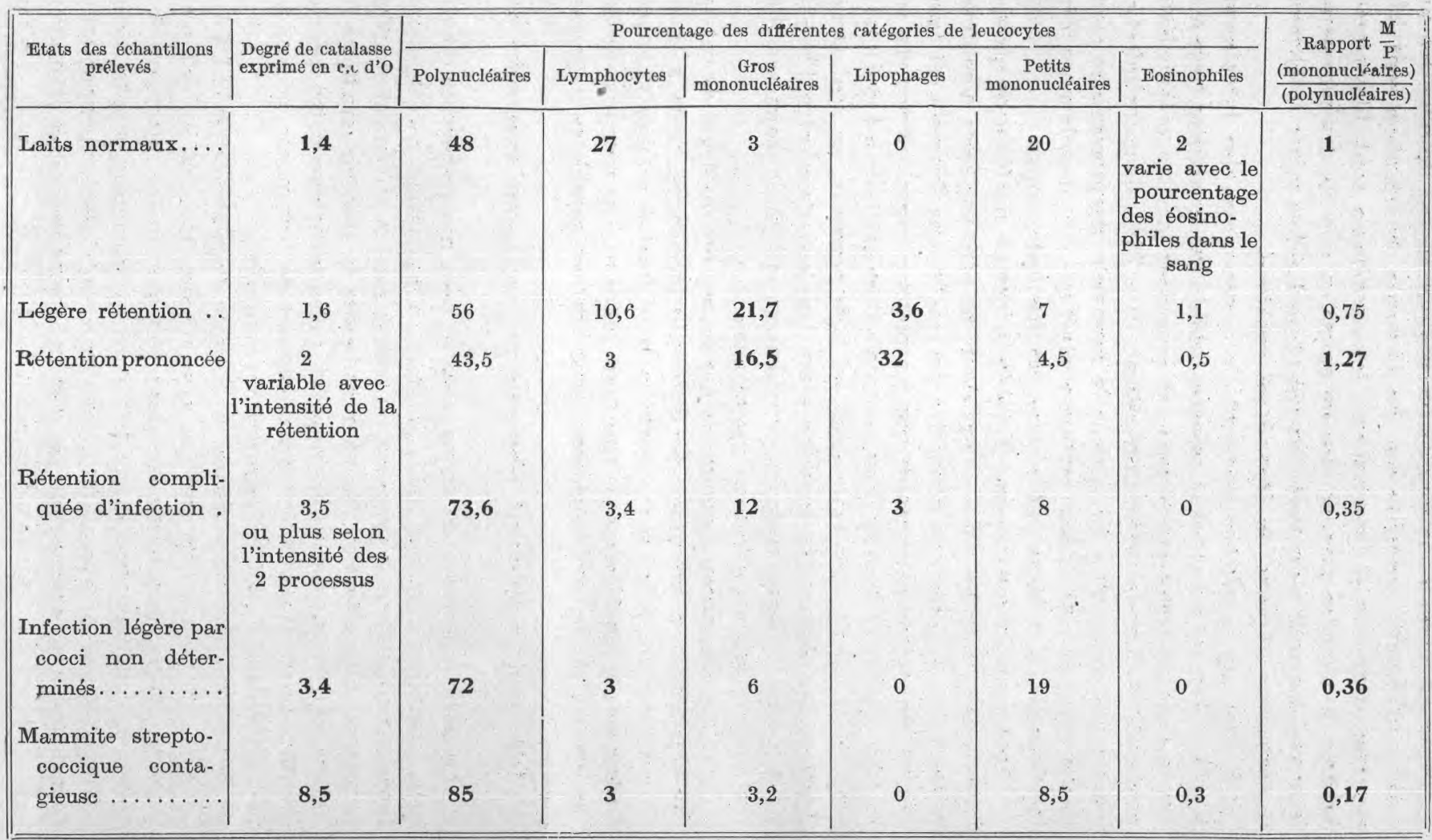


probable que la nature microbienne d'une affection mammaire puisse toujours se traduire par des précisions leucoeytaires quasi-mathématiques; en tous cas, les affections polymicrobiennes, les plus fréquemment rencontrées, ne sauraient se plier à ces exigences. C'est pourquoi nous nous sommes contentés d'envisager le problème à résoudre d'un point de vue plus général en limitant nos investigations au leucocyto-diagnostic différentiel des laits sains, des laits de rétention et des laits d'infection. Nous croyons avoir établi qu'à ces trois catégories de laits correspondent trois types bien différents de formules leucocytaires, de la connaissance desquelles l'expérimentateur averti pourra tirer de précieuses indications tant au point de vue économique qu'au point de vue sanitaire. (Voir les planches en couleurs.)

Puisse surtout cette étude travail contribuer à faire disparaître de la eonsommation sans conditions les laits indésirables et permettre de réserver aux malades et aux enfants les laits de choix auxquels ils ont droit.

\section{BIBLIOGRAPHIE}

Apostoleano (E.). - Contribution à l'étude du système lymphatique mammaire chez les carnivores domestiques. (Thèse de doctorat vétérinaire, Paris, 1925.

Bourgeots (E.-A.-G.). - Rapport sur les leucocytes du lait au VII ${ }^{\mathrm{e}}$ Congrès international de laiterie. (Paris, mai 1926.)

BREED (Robert S.). - The sanitary significance of body cells in milk. (The Journal of Infectious diseases, No 1, January 1914.)

- Cells in milk derived from the udder. (New York Agricultural Experiment Station - Geneva, N. Y. Bulletin No 380 , mars 1914.)

BRo. - Sur l'organisation du contrôle sanitaire et hygiénique du lait. (Revue vétérinaire, 1923, p. 761.)

CAnpbell (H.-C.). - Leucocytes in Milk. Methods of determination and the effect of heat upon their number. (U. S. Department of Agriculture, Bureau of animal industry. Bulletin 117, 18 October 1909.)

Covtrìres (H.). - Biologie médicale, No 6. (juillet-août 1924.)

ERNST (W.): - Grundriss der Milchhygiene für Tierärzte,

JATABERT (E.). - La production laitière à Casablanca: nécessité de son contrôle biologique. Th. Doct.-Vét., Paris, 1925.

Kufferath (H.). - A propos de la recherche des leucocytes dans le lait. (Annales de l'Institut Pasteur, tome XXXIII, 1919, juin No 6.)

Langeron. - Précis de microscopie.

LENZEN (H.). - Uber die Bedeutung und den praktischen Wert der gebraüchlichsten Untersuchungsmethoden der Milch. (Thèse Doc, vét,, Berlin, 1911.)

Marchat (G.). - La leucopédèse gastrique. (Thèse de doctorat, Paris, 1925.)

MARTEu (H.). - Contrôle sanitaire de la production du lait. (Congrès national vétérinaire, 1906.)

- Rapport sur les opérations du service vétérinaire sanitaire de Paris et du département de la Seine, pendant l'année 1910.

Mohler (J.-R.). - Milk and its relation to the public health. (Labor. d'hygiène. Bulletion No 41, E. U. Janvier 1908.) 
NaLLt (V.). - Morphologie des cellules du lait. (Revue d'hygiène et de médecine infantile, janvier 1909.)

NocaRd et LECLATNCHE. - Les maladies microbiennes des animaux. $3^{e}$ édition, 1903.

Panisset (L.). - Insuffisance de l'analyse chimique en face de la nécessité du contrôle bactériologique. (Revue Le Lait, 1921, p. 333.)

Porcher et PANISSEx. - Recherches expérimentales sur le colostrum. (Communication présentée à l'Académie des Sciences, le 17 janvier 1921.)

Porcher (Ch.). - La rétention lactée. (Communication présentée à la Société des Experts-chimistes de France.)

- La réaction de la mamelle à l'infection. Bulletin de la Société des Sciences Vétérinaires de Lyon, 1924.

- Le procès de la matière grasse. (Revue Le Lait, 1925.)

- L'hygiène du lait. (Leçon inaugurale du cours sur le lait. Institut Pasteur, 1923.)

Contrôle dela production du lait.

Rennes. - Le lait qui tue, le lait qui sauve. (Edité par la revue Le Lait, Lyon, 1923.) RoEland. - Aperçu sur le lait à Paris. (Revue Le Lait, 1921, p. 637.)

Rulot (H.). - Intervention des leucocytes dans l'autolyse de la fibrine. (Archives int. de Physiologie, fascicules II et III, juillet 1904, pp. 152-159.)

VARRIER-Jones (P.-C.). - The cellular content of milk : variations met with under physiological and pathological conditions. (The Lancet, 13 septembre 1924.)

Weill et Thévenet. - Des éléments figurés du colostrum et du lait de femme. Archives de Méd. des Enfants, VI, 1903, p. 470.

\section{BIBLIOGRAPHIE ANALYTIQUE}

\section{LES LIVRES}

GARDNER (M.-S.). - L'infirmière visiteuse. 1 vol. broché 461 p. édité par Les Presses Universitaires de France, 49, Boulevard SaintMichel, Paris, 30 fr. 1927.

L'Infirmière visiteuse de Mary Sewall Gardner, directrice du Service des infirmières visiteuses de la ville de Providence, Présidente d'honneur de l'AssociaNationale américaine des Infirmières visiteuses, traduit de l'anglais par Mue Juliette LEFEBvRe et le docteur René SAND, montre d'abord au lecteur l'ori; gine de cette institution, dont la eongrégation des Filles de la Sagesse, due à Saint Vincent-de Paul, a été, si l'on peut dire, le premier modèle, suivi deux siècles plus tard par Miss NightingaL, laquelle après un stage à l'école de Kaiserwerth et chez les Sœurs de Charité à Paris, organisa cette ceuvre en Angleterre.

Sans suivre l'auteur dans tous les plus petits détails de l'organisation des services, de la tâche des divers membres qui les composent, des rapports avec le service médical, toutes choses qui sont fouillées avec le plus grand soin, nous nous attacherons surtout aux services rendus par les infirmières dans la lutte contre les grands maux de l'humanité.

C'est en pénétrant avec tact dans tous les milieux, en devenant l'amie de tous, en faisant prendre les soins non seulement aux malades, mais plus en devinant et prévenant le mal, que l'infirmière contribue pour une large part à sauvegarder des vies humaines et à être un des agents les meilleurs pour diminuer la mortalité. 